\title{
Hydropneumopericardium: Unusual Complication of Proton Radiotherapy
}

\author{
Nakamura Yasuhiro, MD* \\ Department of Gastroenterological Surgery, Toranomon Hospital, Tokyo, Japan
}

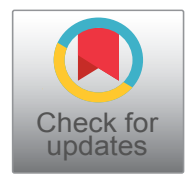

*Corresponding author: Nakamura Yasuhiro, Department of Gastroenterological Surgery, Toranomon Hospital, 2-2-2 Toranomon, Minato Ward, Tokyo, 105-8470, Japan, Tel: +81-3-3588-1111, Fax: +81-3-3582-7068

Hydropnemopericardium is a very rare condition, which arises from penetrating trauma or blunt chest injury, development secondary to procedures, fistulization from adjacent structures and pericardial infections. This patient was 60-year-old man with hepatocellular carcinoma (HCC) presented to outpatients clinic with stomach pain, fatigue and anorexia. The patient was afebrile but laboratory profile manifested an excessive inflammatory response and disseminated intravascular coagulation. We detected a contrast leakage by cholangiogram and abdominal CT showed hydropneumoperi-

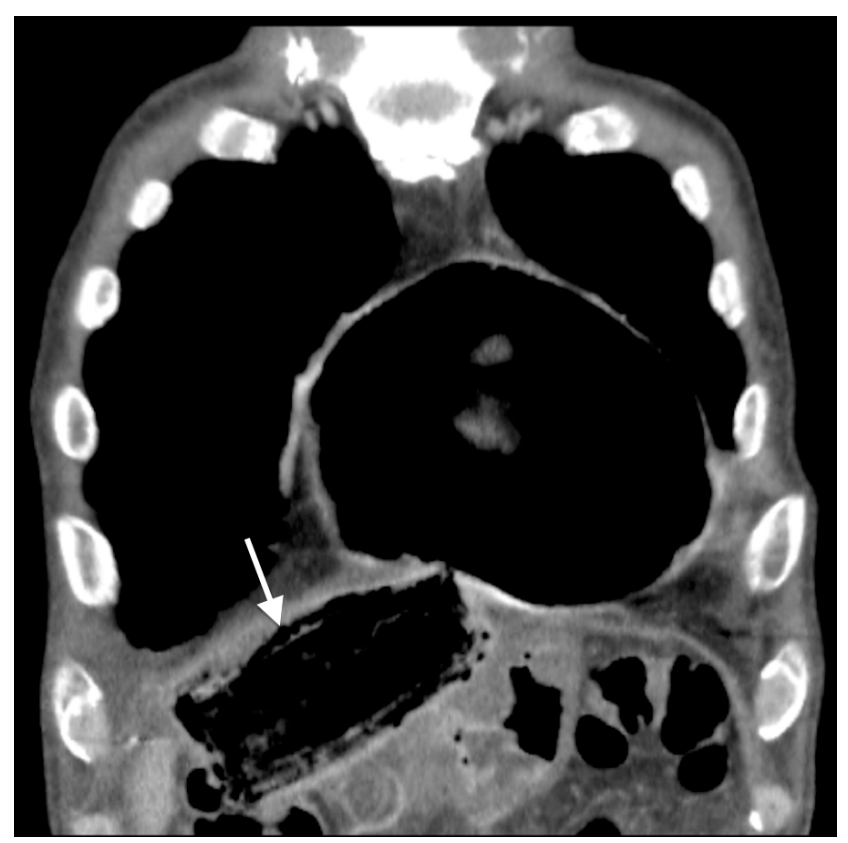

Figure 1: Axial CT image. Pleural effusion in both lung and hydropneumopericardium were confirmed. cardium and low density spacer material in front of liver (Figure 1 and Figure 2). This patient underwent partial hepatectomy and 2-step proton radiotherapy (PRT) with surgical spacer material placement in front of the hepatic hilum for the past 5 years as treatment for HCC. As treatment, we performed surgical pericardiotomy, removal of the spacer material and closed fistulas in diagram, bile duct, duodenum and transverse colon.

\section{Conflicts of Interest}

No conflict of interest to declare.

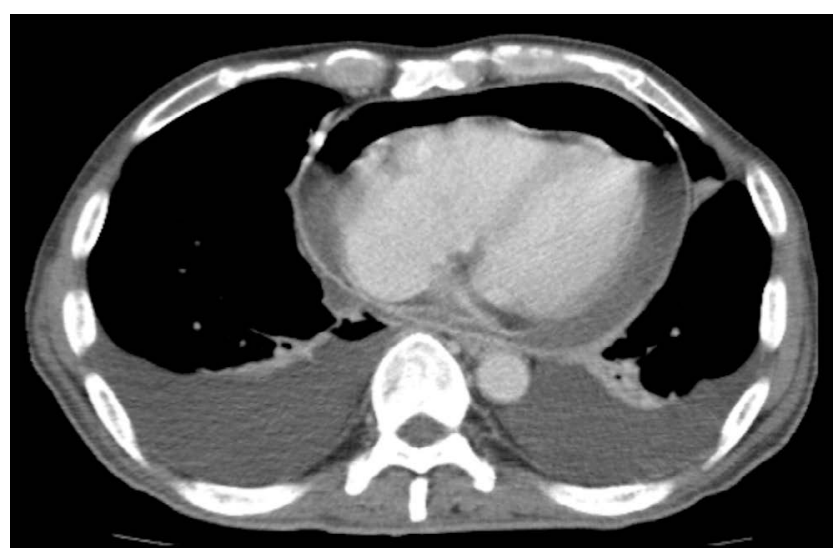

Figure 2: Coronal CT image. Air around heart distend pericardial sac. Spacer material could be seen in front of liver (Arrow). 